\title{
An Actuarial Approach to Reload Option Pricing in Fractional Jump-diffusion Environment
}

\author{
Hong Xue $\mathrm{X}^{1, \mathrm{a}}$, Yonghong $\mathrm{He}^{2, \mathrm{~b}}$ \\ ${ }^{1}$ School of Science, Xi'an Polytechnic University, Xi'an,710048, China \\ ${ }^{2}$ School of Science, Xi'an Polytechnic University, Xi'an,710048, China \\ aemail: xuehonghong@sohu.com, bemail: hyh0802@126.com
}

Keywords: Reload Option; Actuary Method; Jump-diffusion Process; Fractional Brownian Motion

\begin{abstract}
Assume that the underlying asset price follows the fractional jump-diffusion process, the financial market model is built by the stochastic analysis theory for fractional Brownian motion. Using physical probabilistic measure of price process and the principle of fair premium, the pricing formula for reload option is obtained.
\end{abstract}

\section{Introduction}

The reload option is a kind of exotic option. Feng, Liu and Hou (2003) [1] assumed that underlying asset price follows jump-diffusion process in which jumps are constant, and obtained the pricing formula of reload option by martingale method. Wang and Du (2007) [2] assumed that underlying asset price satisfies jump-diffusion process in which the jump process is Poisson process and the height of jump satisfies lognormal distribution, and obtained the pricing formula of reload option by martingale method.

Bladt and Rydberg (1998) [3] proposed the actuarial approach of option price. Using physical probability measure of price process and the principle of fair premium, they deal with the problems of options pricing under the unbalance, arbitrage existing and incomplete circumstance, and transform option pricing into a problem of equivalent and fair insurance premium. Jiao, Liang and Jiang (2009) [4] assumed that the underlying asset price obeys jump-diffusion process, and established the improved reload option pricing model, and obtained the pricing formula of the improved reload option by actuarial approach. Fang, He and Wang (2011) [5] assumed that underlying asset price follows the fractional jump-diffusion process, and obtained the European compound option pricing formula by actuary method.

In this paper, we assume that the underlying asset price follows the fractional jump-diffusion process, and build the financial market mathematical model. Using the stochastic analysis theory for fractional Brownian motion and method for actuarial mathematics, we obtain the reload option pricing formula.

\section{Financial Market Model}

In the financial market, there are two kinds of assets, one is risk-less asset $M_{t}$ which satisfies

$d M_{t}=r M_{t} d t$

Where $r$ is risk-less interest rate; the other is risky asset $S(t)$ which satisfies stochastic differential equation

$$
d S_{t}=S_{t}\left[\mu d t+\sigma d B_{H}(t)+\left(e^{J(t)}-1\right) d Q_{t}\right]
$$

where the expected rate $\mu$ and the volatility rate $\sigma>0$ are constants, $\left\{B_{H}(t), t \geq 0\right\}$ is the fractional Brownian motion on the complete probability space $(\Omega, \mathcal{F}, P), Q_{t}$ is the random jump times of the underlying asset price at the time $[0, t]$, which follows the Poisson process with intensity $\lambda \cdot e^{J(t)}-1$ is the relative jump height of the underlying asset price, and $J(t) \sim N\left(-\sigma_{J}^{2} / 2, \sigma_{J}^{2}\right)$. We assume that $\left\{B_{H}(t), t \geq 0\right\},\{J(t), t \geq 0\}$ and $\left\{Q_{t}, t \geq 0\right\}$ are independent. 
Lemma 1[5] The solution of the stochastic differential equation (2) is $S_{t}=S_{0} \exp \left\{\mu t-\frac{1}{2} \sigma^{2} t^{2 H}+\sigma B_{H}(t)+\sum_{i=1}^{Q_{t}} J(i)\right\}$.

Definition 2[6] The expectation return rate $\beta(u)$ of the $\left\{S_{t}, t \geq 0\right\}$ on $[t, T]$ is defined by $\exp \left\{\int_{t}^{T} \beta(u) d u\right\}=\frac{E\left[S_{T}\right]}{S_{t}}$.

Lemma 3[5] The expectation return rate $\beta(u)$ of the $\left\{S_{t}, t \geq 0\right\}$ on $[t, T]$ satisfies $\beta(u)=\mu$, $u \in[t, T]$

\section{Reload Option Pricing Formula}

Definition 4[7] Consider that the option is reloaded only once before maturity time $T$, the value of reload option at the reloaded time $T_{1}\left(0<T_{1}<T\right)$ is

$V_{T_{1}}=\max \left(S_{T_{1}}-K, 0\right)$,

and the value of reload option at maturity time $T$ is

$$
V_{T}=\left\{\begin{array}{ll}
\frac{K}{S_{T_{1}}} \max \left\{S_{T}-S_{T_{1}}, 0\right\}, & S_{T_{1}}>K, \\
\max \left\{S_{T}-K, 0\right\}, & S_{T_{1}}<K,
\end{array} \quad \text { or } \quad V_{T}= \begin{cases}K \max \left\{\frac{S_{T}}{S_{T_{1}}}-1,0\right\}, & S_{T_{1}}>K, \\
\max \left\{S_{T}-K, 0\right\}, & S_{T_{1}}<K,\end{cases}\right.
$$

where $K$ is strike price, $S_{T_{1}}$ is stock price at reload time $T_{1}, S_{T}$ is stock price at time $T$.

Definition 5 The actuarial price of reload option is defined by

$$
\begin{aligned}
V_{0}= & E\left\{\left\{S_{T_{1}} \exp \left\{-\int_{0}^{T_{1}} \beta(u) d u\right\}-K \exp \left\{-r T_{1}\right\}\right\} I_{A}\right\}+K E\left\{\left\{\left\{S_{T} \exp \left\{-\int_{0}^{T} \beta(u) d u\right\}\right\} /\left\{S_{T_{1}} \exp \left\{-\int_{0}^{T_{1}} \beta(u) d u\right\}-\exp \{-r T\}\right\} I_{A B}\right\}\right. \\
& +E\left\{\left\{S_{T} \exp \left\{-\int_{0}^{T} \beta(u) d u\right\}-K \exp \left\{-\int_{0}^{T} r(u) d u\right\}\right\} I_{C D}\right\},
\end{aligned}
$$

where

$$
\begin{array}{ll}
A=\left\{S_{T_{1}} \exp \left\{-\int_{0}^{T_{1}} \beta(u) d u\right\}>K \exp \left\{-r T_{1}\right\}\right\}, & B=\left\{\left\{S_{T} \exp \left\{-\int_{0}^{T} \beta(u) d u\right\}\right\} /\left\{S_{T_{1}} \exp \left\{-\int_{0}^{T_{1}} \beta(u) d u\right\}>\exp \{-r T\}\right\},\right. \\
C=\left\{S_{T_{1}} \exp \left\{-\int_{0}^{T_{1}} \beta(u) d u\right\}<K \exp \left\{-r T_{1}\right\}\right\}, & D=\left\{S_{T} \exp \left\{-\int_{0}^{T} \beta(u) d u\right\}>K \exp \{-r T\}\right\} .
\end{array}
$$

Theorem 6 The actuarial price of reload option is given by

$$
\begin{aligned}
V_{0}= & \sum_{m=0}^{\infty} \frac{e^{-\lambda T_{1}}\left(\lambda T_{1}\right)^{m}}{m !}\left\{S_{0} \Phi\left(-d^{(m)}+\sigma_{1}^{(m)}\right)-K \exp \left\{-r T_{1}\right\} \Phi\left(-d^{(m)}\right)\right\} \\
& +\sum_{m=0}^{\infty} \sum_{n=0}^{\infty} \frac{e^{-\lambda T}\left(\lambda T_{1}\right)^{m}\left[\lambda\left(T-T_{1}\right)\right]^{n}}{m ! n !}\left\{K \Phi\left(\rho_{1}^{(m, n)} \sigma_{2}^{(n)}-d^{(m)}, \sigma_{2}^{(n)}-a^{(m)}, \rho_{1}^{(m, n)}\right)-K \exp \{-r T\} \Phi\left(-d^{(m)},-a^{(n)}, \rho_{1}^{(m, n)}\right)\right\} \\
& +\sum_{m=0}^{\infty} \sum_{n=0}^{\infty} \frac{e^{-\lambda T}\left(\lambda T_{1}\right)^{m}\left[\lambda\left(T-T_{1}\right)\right]^{n}}{m ! n !}\left\{S_{0} \Phi\left(d^{(m)}-\rho_{2}^{(m, n)} \sigma^{(m, n)}, \sigma^{(m, n)}-c^{(m, n)},-\rho_{2}^{(m, n)}\right)-K \exp \{-r T\} \Phi\left(d^{(m)},-c^{(m, n)},-\rho_{2}^{(m, n)}\right)\right\},
\end{aligned}
$$

where

$$
\begin{array}{ll}
d^{(m)}=\left\{\ln \left(K / S_{0}\right)-r T_{1}+\frac{1}{2} \sigma^{2} T_{1}^{2 H}+m \sigma_{J}^{2} / 2\right\} / \sigma_{1}^{(m)}, & a^{(n)}=\left\{-r T+\frac{1}{2} \sigma^{2}\left(T^{2 H}-T_{1}^{2 H}\right)+n \sigma_{J}^{2}\right\} / \sigma_{2}^{(n)}, \\
c^{(m, n)}=\left\{\ln \left(K / S_{0}\right)-r T_{1}+\frac{1}{2} \sigma^{2} T_{1}^{2 H}+(m+n) \sigma_{J}^{2} / 2\right\} / \sigma^{(m, n)}, & \sigma_{1}^{(m)}=\sqrt{\sigma^{2} T_{1}^{2 H}+m \sigma_{J}^{2}}, \\
\sigma_{2}^{(n)}=\sqrt{\sigma^{2}\left(T^{2 H}-T_{1}^{2 H}\right)+n \sigma_{J}^{2}}, & \sigma^{(m, n)}=\sqrt{\sigma^{2} T^{2 H}+(m+n) \sigma_{J}^{2}}, \\
\rho_{1}^{(m, n)}=\left\{\sigma^{2}\left(T^{2 H}-T_{1}^{2 H}-\left(T-T_{1}\right)^{2 H}\right)\right\} /\left\{2 \sigma_{1}^{(m)} \sigma_{2}^{(n)}\right\}, & \rho_{2}^{(m, n)}=\left\{\sigma^{2}\left(T^{2 H}+T_{1}^{2 H}-\left(T-T_{1}\right)^{2 H}\right)+2 m \sigma_{J}^{2}\right\} /\left\{2 \sigma_{1}^{(m)} \sigma^{(m, n)}\right\}, \\
\varphi(u)=1 / \sqrt{2 \pi} \exp \left\{-u^{2} / 2\right\}, & \varphi(u, v, \rho)=1 /\left\{2 \pi \sqrt{1-\rho^{2}}\right\} \exp \left\{-\left\{u^{2}-2 \rho u v+v^{2}\right\} /\left\{2\left(1-\rho^{2}\right)\right\}\right\}, \\
\Phi(x)=\int_{-\infty}^{x} \varphi(u) d u, & \Phi(x, y, \rho)=\int_{-\infty}^{x} \int_{-\infty}^{y} \varphi(u, v, \rho) d u d v .
\end{array}
$$

Proof: Let

$$
V_{1}=E\left\{\left\{S_{T_{1}} \exp \left\{-\int_{0}^{T_{1}} \beta(u) d u\right\}-K \exp \left\{-r T_{1}\right\}\right\} I_{A}\right\}, \quad V_{2}=E\left\{\left\{S_{T} \exp \left\{-\int_{0}^{T} \beta(u) d u\right\}\right\} /\left\{S_{T_{1}} \exp \left\{-\int_{0}^{T_{1}} \beta(u) d u\right\}-\exp \{-r T\}\right\} I_{A B}\right\},
$$


$V_{3}=E\left\{\left\{S_{T} \exp \left\{-\int_{0}^{T} \beta(u) d u\right\}-K \exp \{-r T\}\right\} I_{C D}\right\}$.

Assume there are the jump $J_{1}(i), i=1,2, \cdots, m$ at $\left[0, T_{1}\right]$, and the jump $J_{2}(i), i=1,2, \cdots, n$ at $\left[T_{1}, T\right]$.

(i) Calculate $V_{1}$. Because

$S_{\bar{T}_{1}}=S_{0} \exp \left\{\mu T_{1}-\frac{1}{2} \sigma^{2} T_{1}^{2 H}-m \sigma_{J}^{2} / 2+\sigma_{1}^{(m)} \xi\right\}, \quad \xi=\left\{\sigma B_{H}\left(T_{1}\right)+\sum_{i=1}^{m} J_{1}(i)+m \sigma_{J}^{2} / 2\right\} /\left\{\sigma_{1}^{(m)}\right\} \sim N(0,1)$,

$A=\left\{S_{T_{1}} \exp \left\{-\int_{0}^{T_{1}} \beta(u) d u\right\}>K \exp \left\{-r T_{1}\right\}\right\}=\left\{\xi>d^{(m)}\right\}$.

\section{Hence}

$$
\begin{aligned}
V_{1} & =E\left\{\left\{S_{T_{1}} \exp \left\{-\int_{0}^{T_{1}} \beta(u) d u\right\}-K \exp \left\{-r T_{1}\right\}\right\} I_{A}\right\}=E\left\{E\left\{\left\{S_{T_{1}} \exp \left\{-\int_{0}^{T_{1}} \beta(u) d u\right\}-K \exp \left\{-r T_{1}\right\}\right\} I_{A} \mid Q_{T_{1}}\right\}\right\} \\
& =\sum_{m=0}^{+\infty} \frac{e^{-\lambda T_{1}}\left(\lambda T_{1}\right)^{m}}{m !} E\left\{\left\{S_{T_{1}} \exp \left\{-\int_{0}^{T_{1}} \beta(u) d u\right\}-K \exp \left\{-r T_{1}\right\}\right\} I_{A} \mid Q_{T_{1}}=m\right\} \triangleq \sum_{m=0}^{+\infty} \frac{e^{-\lambda T_{1}}\left(\lambda T_{1}\right)^{m}}{m !}\left\{V_{11}-V_{12}\right\},
\end{aligned}
$$

where

$$
\begin{aligned}
V_{11} & =E\left\{S_{T_{1}} \exp \left\{-\int_{0}^{T_{1}} \beta(u) d u\right\} I_{A} \mid Q_{T_{1}}=m\right\}=E\left\{S_{0} \exp \left\{-\frac{1}{2} \sigma^{2} T_{1}^{2 H}-m \sigma_{J}^{2} / 2+\sigma_{1}^{(m)} \xi\right\} I_{\left\{\xi>>d^{(m)}\right\}}\right\} \\
& =E\left\{S_{0} \int_{d^{(m)}}^{+\infty} \exp \left\{-\left(\sigma_{1}^{(m)}\right)^{2} / 2+\sigma_{1}^{(m)} u\right\} \frac{1}{\sqrt{2 \pi}} \exp \left\{-\frac{u^{2}}{2}\right\} d u\right\}=S_{0} \int_{d^{(m)}}^{+\infty} \frac{1}{\sqrt{2 \pi}} \exp \left\{-\frac{\left(u-\sigma_{1}^{(m)}\right)^{2}}{2}\right\} d u=S_{0} \Phi\left(-d^{(m)}+\sigma_{1}^{(m)}\right), \\
V_{12} & =E\left\{K \exp \left\{-r T_{1}\right\} I_{A} \mid Q_{T_{1}}=m\right\}=K \exp \left\{-r T_{1}\right\} E\left\{I_{\left\{\xi>d^{(m)}\right\}} \mid Q_{T_{1}}=m\right\}=K \exp \left\{-r T_{1}\right\} P\left\{\xi>d^{(m)}\right\}=K \exp \left\{-r T_{1}\right\} \Phi\left(-d^{(m)}\right) .
\end{aligned}
$$

(ii) Calculate $V_{2}$. Because

$S_{r} / S_{\zeta_{1}}=\exp \left\{\mu\left(T-T_{1}\right)-\frac{1}{2} \sigma^{2}\left(T^{2 H}-T_{1}^{2 H}\right)-n \sigma_{J}^{2} / 2+\sigma_{2}^{(n)} \eta\right\}, \quad \eta=\left\{\sigma\left(B_{H}(T)-B_{H}\left(T_{1}\right)\right)+\sum_{i=1}^{n} J_{2}(i)+n \sigma_{J}^{2} / 2\right\} / \sigma_{2}^{(n)} \sim N(0,1)$,

$$
B=\left\{\frac{S_{T}}{S_{T_{1}}} \exp \left\{-\mu\left(T-T_{1}\right)>\exp \{-r T\}\right\}=\left\{\eta>a^{(n)}\right\}, \quad \rho_{\xi, \eta}=E(\xi \eta)=\rho_{1}^{(m, n)} .\right.
$$

\section{Hence}

$$
\begin{aligned}
V_{2} & =E\left\{\left\{\frac{S_{T}}{S_{T_{1}}} \exp \left\{-\int_{T_{1}}^{T} \beta(u) d u\right\}-\exp \{-r T\}\right\} I_{A B}\right\}=E\left\{E\left\{\left\{\frac{S_{T}}{S_{T_{1}}} \exp \left\{-\int_{T_{1}}^{T} \beta(u) d u\right\}-\exp \{-r T\}\right\} I_{A B} \mid Q_{T_{1}}, Q_{T-T_{1}}\right\}\right\} \\
& =\sum_{m=0}^{+\infty} \sum_{n=0}^{+\infty} \frac{e^{-\lambda T}\left(\lambda T_{1}\right)^{m}\left[\lambda\left(T-T_{1}\right)\right]^{n}}{m ! n !} E\left\{\left\{\frac{S_{T}}{S_{T_{1}}} \exp \left\{-\mu\left(T-T_{1}\right)\right\}-\exp \{-r T\}\right\} I_{\left\{\xi>d^{(m)}, \eta>d^{(m)}\right)} \mid Q_{T_{1}}=m, Q_{T-T_{1}}=n\right\} \\
& =\sum_{m=0}^{+\infty} \sum_{n=0}^{+\infty} \frac{e^{-\lambda T}\left(\lambda T_{1}\right)^{m}\left[\lambda\left(T-T_{1}\right)\right]^{n}}{m ! n !}\left\{V_{21}-V_{22}\right\},
\end{aligned}
$$

where

$$
\begin{aligned}
& V_{21}=E\left\{\frac{S_{T}}{S_{T_{1}}} \exp \left\{-\mu\left(T-T_{1}\right)\right\} I_{\left\{\xi^{\gamma}>d^{(m)}, \eta>a^{(m)}\right\}} \mid Q_{T_{1}}=m, Q_{T-T_{1}}=n\right\}=E\left\{\exp \left\{-\frac{1}{2} \sigma^{2}\left(T^{2 H}-T_{1}^{2 H}\right)-n \sigma_{J}^{2} / 2+\sigma_{2}^{(n)} \eta\right\} I_{\left\{\xi>d^{(m)}, \eta>a^{(m)}\right\}}\right\} \\
& =\exp \left\{-\frac{1}{2}\left(\sigma_{2}^{(n)}\right)^{2}\right\} \int_{d^{(m)}}^{+\infty} \int_{a^{(n)}}^{+\infty} \exp \left\{\sigma_{2}^{(n)} v\right\} \varphi\left(u, v, \rho_{1}^{(m, n)}\right) d u d v=\int_{d^{(m)}}^{+\infty} \int_{a^{(n)}}^{+\infty} \varphi\left(u-\rho_{1}^{(m, n)} \sigma_{2}^{(n)}, v-\sigma_{2}^{(n)}, \rho_{1}^{(m, n)}\right) d u d v
\end{aligned}
$$

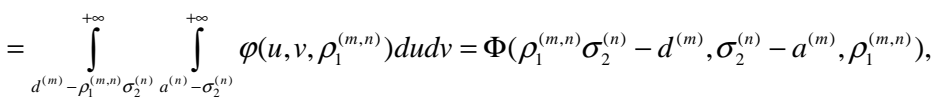

$$
\begin{aligned}
& V_{22}=E\left\{\exp \{-r T\} I_{\left\{\xi>d^{(m)}, \eta>a^{(m)}\right\}} \mid Q_{T_{1}}=m, Q_{T-T_{1}}=n\right\}=\exp \{-r T\} E\left\{I_{\left\{-\xi<-d^{(m)},-\eta<-a^{(n)}\right\}}\right\}=\exp \{-r T\} \Phi\left(-d^{(m)},-a^{(n)}, \rho_{1}^{(m, n)}\right) .
\end{aligned}
$$

(iii) Calculate $V_{3}$. Because

$$
\begin{aligned}
& C=\left\{S_{T_{1}} \exp \left\{-\int_{0}^{T_{1}} \beta(u) d u\right\}<K \exp \left\{-r T_{1}\right\}\right\}=\left\{\xi<d^{(m)}\right\}, \quad S_{T}=S_{0} \exp \left\{\mu T-\frac{1}{2} \sigma^{2} T^{2 H}-(m+n) \sigma_{J}^{2} / 2+\sigma^{(m, n)} \gamma\right\}, \\
& \gamma=\left\{\sigma B_{H}(T)+\sum_{i=1}^{m} J_{1}(i)+\sum_{i=1}^{n} J_{2}(i)+(m+n) \sigma_{J}^{2} / 2\right\} / \sigma^{(m, n)} \sim N(0,1), \\
& D=\left\{S_{T} \exp \left\{-\int_{0}^{T} \beta(u) d u\right\}>K \exp \{-r T\}\right\}=\left\{\gamma>c^{(m, n)}\right\}, \quad \rho_{\xi, \gamma}=E(\xi \gamma)=\rho_{2}^{(m, n)},
\end{aligned}
$$

then

$$
V_{3}=E\left\{\left\{S_{T} \exp \left\{-\int_{0}^{T} \beta(u) d u\right\}-K \exp \{-r T\}\right\} I_{C D}\right\}
$$




$$
\begin{aligned}
& =\sum_{m=0}^{\infty} \sum_{n=0}^{\infty} \frac{e^{-\lambda T}\left(\lambda T_{1}\right)^{m}\left[\lambda\left(T-T_{1}\right)\right]^{n}}{m ! n !} E\left\{\left\{S_{T} \exp \{-\mu T\}\right.\right. \\
& =\sum_{m=0}^{\infty} \sum_{n=0}^{\infty} \frac{e^{-\lambda T}\left(\lambda T_{1}\right)^{m}\left[\lambda\left(T-T_{1}\right)\right]^{n}}{m ! n !}\left\{V_{31}-V_{32}\right\}
\end{aligned}
$$

where

$$
\begin{aligned}
& V_{31}=E\left\{S_{T} \exp \{-\mu T\} I_{\left\{\xi \xi d^{(m)}, \gamma>c^{(m, n)\}}\right\}} \mid Q_{T_{1}}=m, Q_{T-T_{1}}=n\right\}=E\left\{S_{0} \exp \left\{-\frac{1}{2}\left(\sigma^{(m, n)}\right)^{2}+\sigma^{(m, n)} \gamma\right\} I_{\left\{\xi<d^{(m)}, \gamma>c^{(m, n)}\right\}}\right\} \\
& =S_{0} \exp \left\{-\frac{1}{2}\left(\sigma^{(m, n)}\right)^{2}\right\} \int_{-\infty}^{d^{(m)}} \int_{c^{(m, n)}}^{+\infty} \exp \left\{\sigma^{(m, n)} v\right\} \varphi\left(u, v, \rho_{2}^{(m, n)}\right) d u d v=S_{0} \int_{-\infty}^{d^{(m)}} \int_{c^{(m, n)}}^{+\infty} \varphi\left(u-\rho_{2}^{(m, n)} \sigma^{(m, n)}, v-\sigma^{(m, n)}, \rho_{2}^{(m, n)}\right) d u d v \\
& =S_{0} \int_{-\infty}^{d^{(m)}-\rho_{2}^{(m, n)} \sigma^{(m, n)}} \int_{c^{(m, n)}-\sigma^{(m, n)}}^{+\infty} \varphi\left(u, v, \rho_{2}^{(m, n)}\right) d u d v=S_{0} \Phi\left(d^{(m)}-\rho_{2}^{(m, n)} \sigma^{(m, n)}, \sigma^{(m, n)}-c^{(m, n)},-\rho_{2}^{(m, n)}\right), \\
& V_{32}=E\left\{K \exp \{-r T\} I_{\left\{\xi d^{(m)}, \gamma>c^{(m, n)}\right)} \mid Q_{T_{1}}=m, Q_{T-T_{1}}=n\right\}=K \exp \{-r T\} P\left\{\xi<d^{(m)}, \gamma>c^{(m, n)}\right\}=K \exp \{-r T\} \Phi\left(d^{(m)},-c^{(m, n)},-\rho_{2}^{(m, n)}\right) .
\end{aligned}
$$

Remark 7 When the jump intensity $\lambda=0$, we get the actuarial price of reload option in the fractional Brownian motion environment

$$
\begin{aligned}
V_{0}= & S_{0} \Phi\left(-d+\sigma_{1}\right)-K \exp \left\{-r T_{1}\right\} \Phi(-d)+K \Phi\left(\rho_{1} \sigma_{2}-d, \sigma_{2}-a, \rho_{1}\right)-K \exp \{-r T\} \Phi\left(-d,-a, \rho_{1}\right) \\
& +S_{0} \Phi\left(d-\rho_{2} \sigma_{3}, \sigma_{3}-c,-\rho_{2}\right)-K \exp \{-r T\} \Phi\left(d,-c,-\rho_{2}\right),
\end{aligned}
$$

where

$$
\begin{array}{lll}
d=\left\{\ln \left(K / S_{0}\right)-r T_{1}+\frac{1}{2} \sigma^{2} T_{1}^{2 H}\right\} / \sigma_{1}, & a=\left\{-r T+\frac{1}{2} \sigma^{2}\left(T^{2 H}-T_{1}^{2 H}\right)\right\} / \sigma_{2}, & c=\left\{\ln \left(K / S_{0}\right)-r T_{1}+\frac{1}{2} \sigma^{2} T_{1}^{2 H}\right\} / \sigma_{3}, \\
\sigma_{1}=\sigma T_{1}^{H}, & \sigma_{2}=\sigma \sqrt{\left(T^{2 H}-T_{1}^{2 H}\right)}, & \sigma_{3}=\sigma T^{H}, \\
\rho_{1}=\left\{T^{2 H}-T_{1}^{2 H}-\left(T-T_{1}\right)^{2 H}\right\} /\left\{2 T_{1}^{H} \sqrt{\left(T^{2 H}-T_{1}^{2 H}\right)}\right\}, & \rho_{2}=\left\{T^{2 H}+T_{1}^{2 H}-\left(T-T_{1}\right)^{2 H}\right\} /\left\{2 T_{1}^{H} T^{H}\right\} .
\end{array}
$$

\section{Acknowledgement}

In this paper, the research was sponsored by the Scientific Research Program Funded by Shaanxi Provincial Education Department (Program No.12JK0862).

\section{References}

[1] Feng G, Liu Z, and Hou Z. Reload stock options pricing with underlying stock asset obeying jump-diffusion process [J]. Journal of Systemms Engineering, 2003, 18(1) 91-93.

[2] Wang X, Du X. Pricing the reload options under jump-diffusion model [J]. Mathematics in Economics, 2007, 24(3) 276-282.

[3] Bladt M, Rydberg T. An actuarial approach to option pricing under the physical measure and without market assumptions [J]. Insurance: Mathematics and Economics, 1998, 22(1) 65-73.

[4] Jiao L, Liang X, Jiang L. Improved reload option pricing under jump-diffusion model [J]. Journal of Shandong University of Technology, 2009, 23(6) 86-89.

[5] Fang Z, He C, Wang Y. Compound option pricing on fractional jump-diffusions [J]. Mathematics in Practice and Theory, 2011, 41(12) 6-14.

[6] Xue H, Lu J, Li Q, Wang X. Fractional Jump-diffusion Pricing Model under Stochastic Interest Rate [R]. Prceedings 2011 3rd International Conference on Information and Financial Engineering, Shanghai, August, 2011, 428-432.

[7] Fu Q, Shi Z. An actuarial approach fractional geometric Asian-reload stock option and it's simulation [J]. Journal of Chongqing University, 2010, 16(6) 22-25. 